\title{
Verrucomicrobial Methanotrophs
}

\author{
Huub J. M. Op den Camp, Sepehr S. Mohammadi, Arjan Pol, \\ and Peter F. Dunfield
}

\subsection{Methane and Methanotrophy}

The well-studied methanotrophic members of the Alpha- and Gammaproteobacteria have never been found in methane rich environments of extremely low $\mathrm{pH}$. However, methane oxidation activity was detected in geothermal soils characterized by high temperatures $\left(50-95{ }^{\circ} \mathrm{C}\right.$ ) and a $\mathrm{pH}$ as low as 1.0. From 2007 onward, the isolation of new aerobic acidophilic methane oxidizing bacteria from several acidic geothermal ecosystems was reported. They were all identified as new members of the Verrucomicrobia phylum and clustered in the genera Methylacidiphilum (thermophiles) and Methylacidimicrobium (mesophiles). This chapter reports on their discovery, biodiversity, genomics, metabolism, and biotechnological potential.

Methane is released to the atmosphere from natural and anthropogenic sources. The most important sources include natural ecosystems (e.g., wetlands, ruminants, and termites) and anthropogenic activities (e.g., rice paddy fields, landfills, and mining). In these systems, microbial biogenesis of methane is carried out by methanogenic Archaea during organic matter decay under anoxic conditions (Thauer 1998; Conrad 2009; Etiope et al. 2011). In addition, non-microbial methane is emitted to the atmosphere from geothermal regions like cold seeps, mud volcanoes, and fumaroles. This methane is produced primarily via the thermal decomposition of organic matter $\left(>80{ }^{\circ} \mathrm{C}\right)$ in the earth's crust (Etiope and Klusman 2002; Conrad 2009; Etiope et al. 2011). Besides emission sources, sinks of methane are also present on our planet. Atmospheric methane can react with the hydroxyl radical $\mathrm{OH}$ leading to the formation of carbon dioxide and water vapor (Jacob 1999). Methane diffusing from anoxic production zones toward the atmosphere can be

H. J. M. Op den Camp ( $\varangle)$. S. S. Mohammadi · A. Pol Department of Microbiology, IWWR, Radboud University, Nijmegen, The Netherlands e-mail: h.opdencamp@science.ru.nl

P. F. Dunfield

Department of Biological Sciences, University of Calgary, Calgary, Alberta, Canada 
oxidized by both aerobic and anaerobic methane-oxidizing microorganisms, also known as methanotrophs (Forster et al. 2007; Conrad 2009). These microorganisms are assumed to be the major players in keeping the methane balance on our planet by oxidizing 50-80\% of all methane produced before it can reach the atmosphere (Moss et al. 2000; Forster et al. 2007; Conrad 2009).

Microbial oxidation of methane can occur with or without molecular oxygen $\left(\mathrm{O}_{2}\right)$. To date we know that anaerobic oxidation of methane (AOM) can utilize various terminal electron acceptors: sulfate (Knittel and Boetius 2009; Milucka et al. 2012), nitrite (Ettwig et al. 2008), nitrate (Raghoebarsing et al. 2006; Haroon et al. 2013; Arshad et al. 2015), and manganese and iron oxides (Beal et al. 2009; Egger et al. 2015; Scheller et al. 2016). Many other microorganisms can oxidize methane aerobically, with $\mathrm{O}_{2}$ as the terminal electron acceptor. For many years it was believed that aerobic methanotrophy was a phenotype only present in a few bacteria belonging to the phylum Proteobacteria, specifically in the classes Alphaproteobacteria (also named type II methanotrophs) and Gammaproteobacteria (type I methanotrophs) (Hanson and Hanson 1996; Dedysh et al. 2000; Op den Camp et al. 2009). The two groups differ in their pathways for biomass production, the ribulose monophosphate (RuMP) pathway in the Gammaproteobacteria versus the serine pathway in the Alphaproteobacteria (Hanson and Hanson 1996; Chistoserdova et al. 2009; Chistoserdova 2011). Other broad physiological and structural characteristics separating the two groups (cell shape, ultrastructure of intracytoplasmic membranes, the main phospholipid fatty acids, nitrogen fixation ability, presence of different monooxygenases, etc.) were also defined in the early years of research into these bacteria (Hanson and Hanson 1996), but recently many genera have been discovered that do not fit these sweeping generalizations (e.g., Methylocella, Methylohalobius, Methylovulum, and Methyloceanibacter; Dedysh et al. 2000; Heyer et al. 2005; Iguchi et al. 2011; Vekeman et al. 2016).

\subsection{The Discovery of the Aerobic Verrucomicrobial Methanotrophs}

The methanotrophic members of the Alpha- and Gammaproteobacteria have never been found in methane-rich environments of extremely low $\mathrm{pH}$ (Op den Camp et al. 2009). The most acidophilic proteobacterial methanotrophs known, species of Methylocella and Methylocapsa, grow in peat environments at $\mathrm{pHs}$ as low as 4.2 (Dedysh et al. 1998, 2002; Dunfield and Dedysh 2010). However, methane oxidation activity was detected in geothermal soils in the Solfatara at Pozzuoli near Naples (Italy), a site characterized by high temperatures $\left(50-95^{\circ} \mathrm{C}\right)$ and a $\mathrm{pH}$ as low as 1.0 (Castaldi and Tedesco 2005). The hydrogen sulfide present in the hot fumarolic gas at this site is oxidized into sulfuric acid biotically or abiotically, thereby forming an extremely acidic ecosystem. Biogeochemical evidence for methane oxidation at extremely low $\mathrm{pH}$ was confirmed in late 2007 to early 2008 by three independent studies that obtained isolates of new aerobic methane-oxidizing bacteria from several acidic volcanic samples from the Solfatara; from Hell's Gate, Tikitere (New Zealand); and from the Uzon Caldera, Kamchatka (Russia) (Pol et al. 2007; 


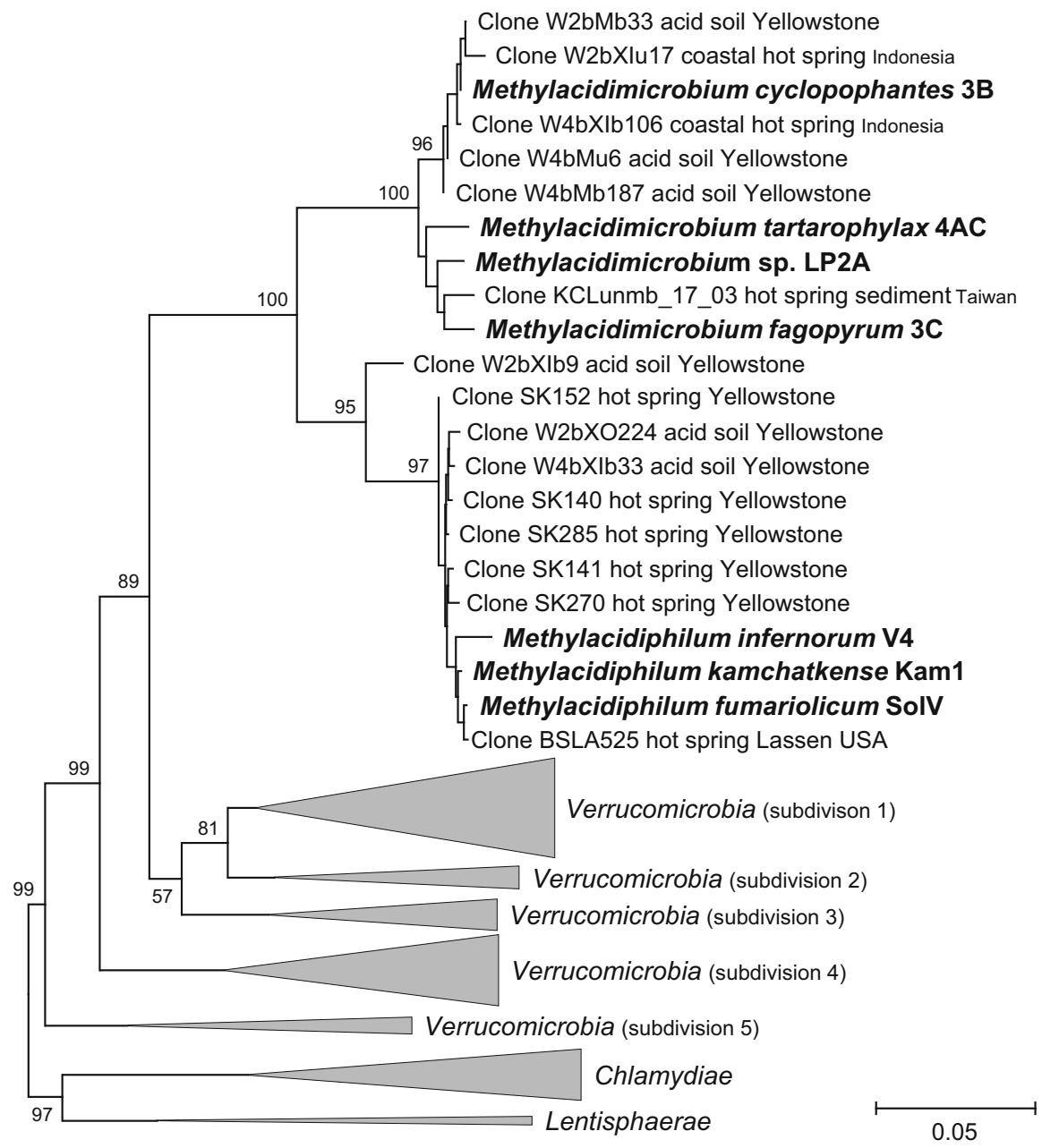

Fig. 3.1 Phylogenetic tree of 16S rRNA gene sequences of methanotrophic and other Verrucomicrobia showing the evolutionary relationships of the methanotrophic mesoacidophilic Methylacidimicrobium strains (3B, 3C, LP2A, and 4AC), the methanotrophic thermoacidophilic Methylacidiphilum strains (V4, SolV, and Kam1), and other members of the phylum Verrucomicrobia and other selected phyla. Cultivated representatives are indicated in boldface. Chlamydiae and Lentisphaerae species were used as outgroup

Dunfield et al. 2007; Islam et al. 2008). All three isolates were able to grow at $\mathrm{pH}$ 1 and temperatures up to $65^{\circ} \mathrm{C}$ (Dunfield et al. 2007; Pol et al. 2007; Islam et al. 2008; Op den Camp et al. 2009). Interestingly, based on 16S ribosomal RNA gene analysis, all three strains (SolV, V4, and Kam1) were identified as members of the Verrucomicrobia phylum and phylogenetically formed a single genus-level cluster for which the name Methylacidiphilum was suggested (Fig. 3.1) (Op den Camp et al. 2009). This genus has yet to be taxonomically validated because of difficulties in maintaining a viable culture in reference culture collections, but is a useful label for 
consistent identification. The link between a member of the widely distributed Verrucomicrobia phylum and a major global process like the methane cycle was an exciting discovery. Despite the importance of Verrucomicrobia in many different ecosystems, which is suggested by their abundance in 16S rRNA amplicons from environmental DNA extracts, the portion of cultivated representatives within the Verrucomicrobia is quite low (Hugenholtz et al. 1998). 16S rRNA gene phylogenetic analyses have allowed a division of the phylum into five subdivisions (Fig. 3.1), but the physiology of the different subdivisions is still poorly understood (Wagner and Horn 2006).

\subsection{Diversity of Verrucomicrobia methanotrophs}

After the discovery of the thermoacidophilic genus Methylacidiphilum, van Teeseling et al. (2014) and Sharp et al. (2014) isolated, characterized, and described four new species of mesophilic acidophilic verrucomicrobial methanotrophs from low-temperature geothermal sites: the soil of the Solfatara crater, which is at the center of the Campi Flegrei caldera, near Naples (Italy), and a geothermally impacted soil in Reporoa, New Zealand. The 16S rRNA genes of these new isolates were very similar to each other, but were less than $90 \%$ identical to those of the Methylacidiphilum species described earlier. Furthermore, PmoA- and MxaF-/XoxFbased phylogenies showed similar clustering compared to the 16S rRNA-based phylogeny (Keltjens et al. 2014; van Teeseling et al. 2014). The new genus name Methylacidimicrobium was proposed for these mesophilic verrucomicrobial methanotrophs, including the species Methylacidimicrobium tartarophylax 4AC, Methylacidimicrobium fagopyrum 3C, Methylacidimicrobium cyclopophantes 3B, and Methylacidimicrobium sp. LP2A (van Teeseling et al. 2014). Again these names are not yet taxonomically validated.

Together, the thermophilic and mesophilic methanotrophs described form a coherent class-level clade within the Verrucomicrobia phylum (Fig. 3.1). 16S rRNA gene sequences belonging to this group have since been detected in other acidic geothermal habitats of Europe, Asia, and North America, indicating their widespread occurrence in such systems (Kozubal et al. 2012; Li et al. 2016; Gagliano et al. 2014). In an extensive survey of environments in Canada and New Zealand using highthroughput sequencing of 16S rRNA amplicons, Sharp et al. (2014) found putative methanotrophic Verrucomicrobia in samples covering a broad temperature range $\left(22.5-81.6{ }^{\circ} \mathrm{C}\right)$, but only under acidic conditions $(\mathrm{pH}<5.0)$ and only in geothermal systems. They could not be detected in acidic non-geothermal methane-rich environments like fens and bogs, suggesting that factors other than $\mathrm{pH}$ alone also affect their distribution. However, similar $16 \mathrm{~S}$ rRNA sequences have recently been detected in highly acidic microbially induced concrete corrosion zones of sulfideimpacted sewage pipes (Pagaling et al. 2014). This suggests that methanotrophic Verrucomicrobia are indeed present in some non-geothermal ecosystems, at least $\mathrm{H}_{2} \mathrm{~S}$-rich ones. The factors controlling the ecological range of these bacteria are not yet completely understood, but may be related to their rare earth metal requirement (see below) in addition to their preference for highly acidic conditions. 
Verrucomicrobial methanotrophs

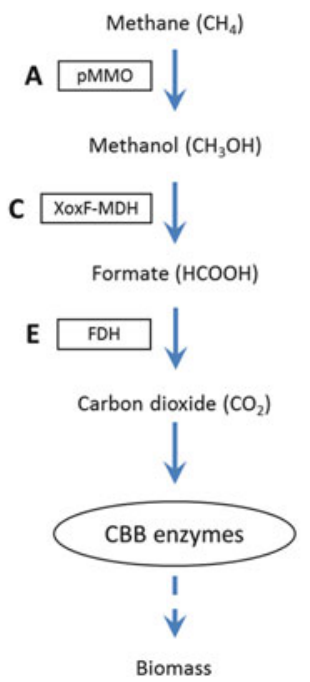

Proteobacterial methanotrophs

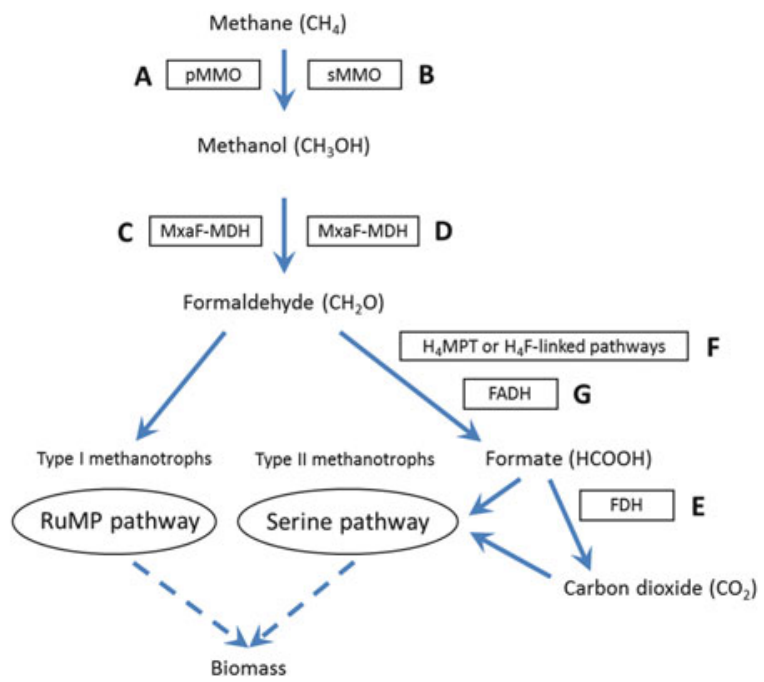

Fig. 3.2 Methane oxidation pathways in verrucomicrobial (left) and proteobacterial (right) methanotrophs with all enzymes (boxes) involved. (A) Particulate methane monooxygenase (pMMO); (B) soluble methane monooxygenase (sMMO). (C) Lanthanide-dependent methanol dehydrogenase (XoxF-MDH); (D) calcium-dependent methanol dehydrogenase (MxaF-MDH); (E) formate dehydrogenase (FDH); (F) multienzyme cofactor-linked $\mathrm{C} 1$ transfer pathways $\left(\mathrm{H}_{4} \mathrm{MPT}=\right.$ tetrahydromethanopterin, $\mathrm{H}_{4} \mathrm{~F}=$ tetrahydrofolate $)$; $(\mathbf{G})$ formaldehyde dehydrogenase (FADH). Formaldehyde is assimilated in type I proteobacterial methanotrophs via the ribulose monophosphate (RuMP), and formate is the main branch point to the serine pathways in type II proteobacterial methanotrophs. In verrucomicrobial methanotrophs, carbon dioxide is fixed using the Calvin-Benson-Bassham (CBB) cycle enzymes

\subsection{Genomics and Metabolism of Verrucomicrobia Methanotrophs}

The aerobic oxidation of methane has the net reaction, $\mathrm{CH}_{4}+2 \mathrm{O}_{2} \rightarrow \mathrm{CO}_{2}+2 \mathrm{H}_{2} \mathrm{O}$ $\left(\Delta \mathrm{G}^{\circ}=-773 \mathrm{KJ}\right.$ per mol $\left.\mathrm{CH}_{4}\right)$, but proceeds microbially via the intermediates methanol $\left(\mathrm{CH}_{3} \mathrm{OH}\right)$, formaldehyde $\left(\mathrm{CH}_{2} \mathrm{O}\right)$, and formate $(\mathrm{CHOOH})(\mathrm{Chistoserdova}$ et al. 2009; Hanson and Hanson 1996) (Fig. 3.2). In this reaction scheme, electrons from methane are transported to a membrane-bound electron transport chain using a pyrroloquinoline quinone cofactor to cytochrome $c$ (methanol dehydrogenase) or NAD (in formaldehyde oxidation systems and formate dehydrogenase). The terminal electron acceptor in aerobic methane oxidation is $\mathrm{O}_{2}$.

The first step in methane oxidation is catalyzed by the methane monooxygenase enzyme (MMO) converting methane into methanol. This enzyme exists in two distinct forms; the soluble cytoplasmic form (sMMO, NADH-dependent) and the particulate membrane-associated form (pMMO, cytochrome $c$ dependent) (Hanson and Hanson 1996; Fig. 3.2). In general, sMMO is located in the cytoplasm, and pMMO is situated in 
the intracytoplasmic membranes (ICMs) present in many proteobacterial methanotrophs (Nguyen et al. 1998; Brantner et al. 2002). The soluble cytoplasmic methane monooxygenase (sMMO) is only present in a limited number of methanotrophs, usually in addition to pMMO (Chistoserdova 2011), but occasionally as the sole MMO (Methylocella, Methyloferula, and Methyloceanibacter) has only the sMMO enzyme (Dedysh et al. 2005; Dunfield and Dedysh 2010; Vekeman et al. 2016). The completed circular genomes of $M$. infernorum strain V4 and $M$. fumariolicum strain SolV lack the genes encoding for sMMO subunits (Pol et al. 2007; Hou et al. 2008; Op den Camp et al. 2009; Anvar et al. 2014). Draft genomes of M. kamchatkense (Kam1) and the mesophilic Methylacidimicrobium strains also lack sMMO-encoding genes (Erikstad and Birkeland 2015; Sharp et al. 2014; van Teeseling et al. 2014). On the other hand, complete $p m o C A B$ operons encoding pMMO are found in the genome of every strain. The Methylacidimicrobium strains either contain a single pmoCAB operon (van Teeseling et al. 2014) or in the case of strain LP2A two nearly identical operons and an orphan third $p m o C$ copy (Sharp et al. 2014). The completed thermoacidophilic Methylacidiphilum genomes each contain three complete pmoCAB operons and an orphan fourth pmoC copy (Op den Camp et al. 2009), while the draft genome of $M$. kamchatkense strain Kam1 showed three complete pmoCAB operons and a fourth operon without the $p m o B$ gene (Op den Camp et al. 2009; Erikstad et al. 2012). Remarkably, the three paralogous operons in Methylacidiphilum are phylogenetically divergent from one another by up to $50 \%$ amino acid sequence. Alien Hunter, a program that uses nucleotide k-mer frequencies to identify potential lateral gene transfer (Vernikos and Parkhill 2006 ), predicts that the pmoCAB3 operon, the most divergent of the three, has been obtained by lateral gene transfer from another organism (Sharp et al. 2013). However, as no other organism is known with a similar operon, the source of this transfer is unknown.

This presence of diverse pmo copies in the Methylacidiphilum genomes is not unique, as some proteobacterial methanotrophs also possess multiple, divergent copies of pmo operons. A divergent pmoCAB2 was reported to be present in many type II proteobacterial methanotrophs, including the model strain Methylocystis SC2 (Tchawa Yimga et al. 2003). Baani and Liesack (2008) reported that the enzymes encoded by the two paralogous operons in this model strain showed different apparent $\mathrm{K}_{\mathrm{m}}$ values. Recently, a sequence-divergent pmo (named pxm) was also found in some type I proteobacterial methanotrophs (Tavormina et al. 2011). The existence of sequence-divergent copies may indicate different physiological functions under different environmental conditions. In Methylacidiphilum species, the functions of the three paralogous operons are not yet clear, although initial expression studies have been performed. Khadem et al. (2012a) showed that pmoCAB1 and pmoCAB2 operons of strain SolV are highly but differentially expressed under oxygen limitation and oxygen excess, respectively. Interestingly, the third $p m o C A B$ operon ( $p m o C A B 3$ ) of strain SolV was not expressed under any of conditions tested (Khadem et al. 2012a). In M. kamchatkense strain Kam1, growth on methanol instead of methane resulted in a downregulation of all pmoA genes (Erikstad et al. 2012). 
The second enzyme involved in the aerobic oxidation of methane is the PQQ-dependent methanol dehydrogenase (MDH) converting methanol into formaldehyde (Fig. 3.2). This enzyme includes a large and a small subunit encoded by mxaFI genes and needs pyrroloquinoline quinone as cofactor and a cytochrome $c$ electron acceptor encoded by the $m x a G$ gene. Remarkably, in all available verrucomicrobial aerobic methanotrophic strains (both Methylacidiphilum and Methylacidimicrobium spp.), the well-studied mxaFJGIRSACKLDEHB cluster encoding the canonical mxaF-type MDH was absent and substituted by xoxFGJ, while biogenesis of the cofactor pyrroloquinoline quinone is encoded by pqqABCDEF (Hou et al. 2008; Op den Camp et al. 2009; Pol et al. 2014; Keltjens et al. 2014; van Teeseling et al. 2014; Sharp et al. 2014). After the observation that growth of $M$. fumariolicum SolV was strictly depended on the addition of mudpot water from its natural environment, it was discovered that the purified XoxG-type $\mathrm{MDH}$ of M. fumariolicum strain SolV contains lanthanides at the catalytic site, while previously studied mxaF-type MDHs have calcium as active metal at this site (Pol et al. 2014; Keltjens et al. 2014). It was also shown that the growth of strain SolV is dependent on lanthanides at submicromolar concentrations, which could replace the mudpot water.

The presence of a lanthanide in the PQQ catalytic center seems to make XoxFtype MDHs more efficient catalysts in methanol conversion, since they efficiently oxidize not only methanol but also formaldehyde. The oxidation of methanol into formate may have major implications for the diversity of methylo- and methanotrophic catabolism and anabolism. The field of methano- and methylotrophy is a rapidly expanding puzzle of redundant anabolic and catabolic possibilities and opportunities (Chistoserdova et al. 2009; Chistoserdova 2011).

The third step in the proteobacterial aerobic oxidation of methane is the conversion of formaldehyde to formate. Formaldehyde is a toxic intermediate and must be maintained at nontoxic levels (Chistoserdova 2011). Different formaldehydeoxidizing systems are present in methylotrophs (Chistoserdova 2011). Formaldehyde oxidation can be carried out by a formaldehyde dehydrogenase (FADH; Fig. 3.2). This single enzyme is linked to NAD or mycothiol (Chistoserdova 2011). In addition, formaldehyde oxidation can be performed by multienzyme cofactor-linked $\mathrm{C} 1$ transfer pathways. Two important pathways for formaldehyde oxidation in methylotrophs require $\mathrm{H}_{4} \mathrm{MPT}$ (tetrahydromethanopterin) or $\mathrm{H}_{4} \mathrm{~F}$ (tetrahydrofolate) as cofactors (Chistoserdova et al. 2009; Chistoserdova 2011). The $\mathrm{H}_{4}$ MPT pathway is common in proteobacterial methanotrophs but appears to be missing from the genomes of all the methanotrophic Verrucomicrobia. Some parts of a $\mathrm{H}_{4} \mathrm{~F}$ pathway are present, but this pathway has not been verified. $M$. fumariolicum strain SolV lacks FADH as well as $\mathrm{H}_{4}$ MPT pathway-encoding genes, but the lanthanide-dependent XoxF-type MDH was shown to oxidize methanol directly to formate (see above), so a specialized formaldehyde oxidation system may not be needed by these methanotrophs.

The last step in methane oxidation is the conversion of formate into carbon dioxide by formate dehydrogenase (FDH; Fig. 3.2). The complete genome of strains V4 and SolV and the draft genome of strain Kam1 show that formate oxidation is 
probably conducted by a NAD-dependent formate dehydrogenase and a membranebound formate dehydrogenase (Pol et al. 2007; Hou et al. 2008; Anvar et al. 2014; Erikstad and Birkeland 2015). In proteobacterial methanotrophs, formaldehyde and/or formate is assimilated via two main pathways: the RuMP and serine pathways which are important for Gammaproteobacteria (type I) and Alphaproteobacteria (type II) methanotrophs, respectively (Hanson and Hanson 1996; Chistoserdova et al. 2009). In the RuMP pathway, the unique enzymes hexulose-6-phosphate synthase and hexulose-6-phosphate isomerase catalyze the reactions to assimilate formaldehyde to form glyceraldehyde-3-phosphate as an intermediate (Hanson and Hanson 1996). In the serine pathway, the exclusive reactions are catalyzed by serine hydroxymethyltransferase (STHM), hydroxypyruvate reductase (HPR), malate thiokinase (MTK), and malyl coenzyme A lyase (MCL) (Hanson and Hanson 1996). In this pathway, both formate and carbon dioxide are utilized to produce acetyl coenzyme A for biosynthesis (Crowther et al. 2008; Šmejkalová et al. 2010). Based on the full genomes of the verrucomicrobial methanotrophs strain SolV and V4, and the transcriptome data of strain SolV, it is clear that these bacteria do not follow either the RuMP or the serine pathway (Hou et al. 2008; Op den Camp et al. 2009; Anvar et al. 2014). Rather, the verrucomicrobial methanotrophs are autotrophs, fixing carbon from carbon dioxide using the Calvin-Benson-Bassham (CBB) cycle (Khadem et al. 2011; Sharp et al. 2012). Recently, Rasigraf et al. (2014) also showed autotrophic carbon dioxide fixation via the Calvin-Benson-Bassham cycle by the denitrifying methanotroph Methylomirabilis oxyfera belonging to the NC10 phylum.

In addition to methane oxidation, M. fumariolicum strain SolV is able to fix $\mathrm{N}_{2}$ at low oxygen concentrations using an extremely oxygen-sensitive nitrogenase (Khadem et al. 2010). Furthermore, the storage of carbon in strain SolV was demonstrated. Glycogen was formed in the cells once ammonium was depleted and methane still present (Khadem et al. 2012b). Gammaproteobacterial methanotrophs may also produce glycogen, while alphaproteobacterial methanotrophs have been shown to produce PHB (polyhydroxybutyric acid) as a storage compound (Linton and Cripps 1978; Pieja et al. 2011a, b; Eshinimaev et al. 2002).

\subsection{Knallgas Bacteria}

Recently, it was shown that the methanotroph Methylacidiphilum fumariolicum SolV could also grow as a real "Knallgas" bacterium consuming hydrogen/carbon dioxide in the absence of methane (Mohammadi et al. 2017). Two sets of hydrogen uptake hydrogenase genes were identified in the genome of strain SolV, encoding an oxygen-insensitive (hhy-type) and an oxygen-sensitive (hup-type) enzyme. Transcriptome analysis revealed that the hhy-type hydrogenase was constitutively expressed and active. This hydrogenase was supposed to support growth on hydrogen only at oxygen concentrations below $1.5 \%$. When oxygen was further educed to 
below $0.2 \%$, expression of the oxygen-sensitive hup-type hydrogenase was induced, and the growth rate increased to about $60 \%$ of the rate on methane.

In an independent study, Carere et al. (2017) showed that the environmental isolate Methylacidiphilum sp. RTK17.1 sustained aerobic respiration and carbon fixation using methane and hydrogen as electron donors either in concert or separately depending on substrate availability. This lifestyle may have facilitated expansion of their niche space in geothermally influenced surface soils.

Taken together, these results suggest that in their natural environments, where both hydrogen and methane might be limiting, verrucomicrobial methanotrophs may operate primarily as "Knallgas" bacteria or prefer a mixotrophic lifestyle. In view of these findings, the role of hydrogen in methanotrophic ecosystems has to be revised, especially in soil, and related to consumption of atmospheric methane.

\subsection{Biotechnology}

The low cost of natural gas and biogas compared to other fuels makes them promising feedstocks for bioconversions. Possible bioproducts include polymers, single-cell protein, vitamins, carotenoids, compatible solutes, lipids, or methanol (Strong et al. 2015). As of yet, there are no published reports of the use of verrucomicrobial methanotrophs for bioconversion or indeed for any other biotechnological use. At the moment one can only speculate that their unique biochemistry compared to all other methanotrophs may present unique possibilities for bioproduct discovery and production.

Ecologically, the Verrucomicrobia methanotrophs occupy a niche separate from proteobacterial methanotrophs and may therefore also present unique possibilities for environmental biotechnology. For example, biofiltration of methane is a useful way of reducing the carbon footprint of waste gas streams that are economically not feasible to recover because they are intermittent, remote, and contaminated or have low flow rates or low methane contents (Hettiarachchi and Hettiaratchi 2011). Via biofiltration, the potent greenhouse gas methane is oxidized to $\mathrm{CO}_{2}$, a gas with a global warming potential 34 times less than methane. The presence of $\mathrm{H}_{2} \mathrm{~S}$ in biogas or natural gas ("sour gas") is a general problem for the sustained operation of a methane biofilter and in some situations is also problematic for the economic recovery of fuel methane. The Verrucomicrobia methanotrophs are naturally adapted to sour gas sources and may therefore be useful in biofiltration of sour gas. Their unique tolerance may even make sour gas streams viable feedstocks for biotechnology without a desulfurization step. The observed co-occurrence of sulfur- and methane-oxidizing acidophiles in soured pipes (Pagaling et al. 2014) indicates the possibility for simultaneous $\mathrm{H}_{2} \mathrm{~S}$ and $\mathrm{CH}_{4}$ removal from waste gas. 


\section{References}

Anvar SY, Frank J, Pol A et al (2014) The genomic landscape of the verrucomicrobial methanotroph Methylacidiphilum fumariolicum SolV. BMC Genomics 15:914

Arshad A, Speth D, de Graaf R et al (2015) A metagenomics-based metabolic model of nitratedependent anaerobic oxidation of methane by Methanoperedens-like archaea. Front Microbiol 6:1423

Baani M, Liesack W (2008) Two isozymes of particulate methane monooxygenase with different methane oxidation kinetics are found in Methylocystis sp. strain SCZ. Proc Natl Acad Sci USA 105:10203-10208

Beal EJ, House CH, Orphan VJ (2009) Manganese- and iron-dependent marine methane oxidation. Science 325:184-187

Brantner CA, Remsen CC, Owen HA et al (2002) Intracellular localization of the particulate methane monooxygenase and methanol dehydrogenase in Methylomicrobium album BG8. Arch Microbiol 178:59-64

Carere CR, Hards K, Houghton KM et al (2017) Mixotrophy drives niche expansion of verrucomicrobial methanotrophs. ISME J 11:2599-2610

Castaldi S, Tedesco D (2005) Methane production and consumption in an active volcanic environment of Southern Italy. Chemosphere 58:131-139

Chistoserdova L, Kalyuzhnaya MG, Lidstrom ME (2009) The expanding world of methylotrophic metabolism. Annu Rev Microbiol 63:477-499

Chistoserdova L (2011) Modularity of methylotrophy, revisited. Environ Microbiol 13:2603-2622

Conrad R (2009) The global methane cycle: recent advances in understanding the microbial processes involved. Environ Microbiol Rep 1:285-292

Crowther GJ, Kosály G, Lidstrom ME (2008) Formate as the main branch point for methylotrophic metabolism in Methylobacterium extorquens AM1. J Bacteriol 190:5057-5062

Dedysh SN, Panikov NS, Liesack W et al (1998) Isolation of acidophilic methane-oxidizing bacteria from northern peat wetlands. Science 282:281-284

Dedysh SN, Liesack W, Khmelenina VN et al (2000) Methylocella palustris gen. nov., sp. nov., a new methane-oxidizing acidophilic bacterium from peat bags, representing a novel subtype of serine-pathway methanotrophs. Int J Syst Evol Microbiol 50:955-969

Dedysh SN, Khmelenina VN, Suzina NE et al (2002) Methylocapsa acidiphila gen. nov., sp. nov., a novel methane-oxidizing and dinitrogen-fixing acidophilic bacterium from Sphagnum bog. Int J Syst Evol Microbiol 52:251-261

Dedysh SN, Knief C, Dunfield PF (2005) Methylocella species are facultatively methanotrophic. J Bacteriol 187:4665-4670

Dunfield PF, Dedysh SN (2010) Acidic methanotrophic environments. In: Timmis KN (ed) Handbook of hydrocarbon and lipid microbiology. Springer-Verlag, Berlin, pp 2181-2192

Dunfield PF, Yuryev A, Senin P et al (2007) Methane oxidation by an extremely acidophilic bacterium of the phylum Verrucomicrobia. Nature 450:879-883

Egger M, Rasigraf O, Sapart CJ et al (2015) Iron-mediated anaerobic oxidation of methane in brackish coastal sediments. Environ Sci Technol 49:277-283

Erikstad HA, Jensen S, Keen TJ et al (2012) Differential expression of particulate methane monooxygenase genes in the verrucomicrobial methanotroph 'Methylacidiphilum kamchatkense' Kam1. Extremophiles 16:405-409

Erikstad HA, Birkeland NK (2015) Draft genome sequence of "Candidatus Methylacidiphilum kamchatkense" strain Kam1, a thermoacidophilic methanotrophic Verrucomicrobium. Genome Announc 3:e00065-15

Eshinimaev BT, Khmelenina VN, Sakharovskiı̆ VG et al (2002) Physiological, biochemical, and cytological characteristics of a haloalkalitolerant methanotroph grown on methanol. Microbiology 71:512-518 
Etiope G, Klusman RW (2002) Geologic emissions of methane to the atmosphere. Chemosphere 49:777-789

Etiope G, Oehler DZ, Allen CC (2011) Methane emissions from Earth's degassing: implications for Mars. Planet Space Sci 59:182-195

Ettwig KF, Shima S, van de Pas-Schoonen KT et al (2008) Denitrifying bacteria anaerobically oxidize methane in the absence of Archaea. Environ Microbiol 10:3164-3173

Forster P, Ramaswamy V, Artaxo P et al (2007) Changes in atmospheric constituents and in radiative forcing. In: Solomon $S$ et al (eds) Climate change 2007: the physical science basis. Contribution of working group I to the fourth assessment report of the intergovernmental panel on climate change. Cambridge University Press, UK, pp 129-234

Gagliano AL, D'Alessandro W, Tagliavia M et al (2014) Methanotrophic activity and diversity of methanotrophs in volcanic geothermal soils at Pantelleria (Italy). Biogeosciences 11:5865-5875

Hanson RS, Hanson TE (1996) Methanotrophic bacteria. Microbiol Rev 60:439-471

Haroon MF, Hu S, Shi Y et al (2013) Anaerobic oxidation of methane coupled to nitrate reduction in a novel archaeal lineage. Nature 500:567-570

Hettiarachchi V, Hettiaratchi P (2011) Field-scale operation of methane biofiltration systems to mitigate point source methane emissions. Environ Pollut 159:1715-1720

Heyer J, Berger U, Hardt M, Dunfield PF (2005) Methylohalobius crimeensis gen. nov., sp. nov., a moderately halophilic, methanotrophic bacterium isolated from hypersaline lakes of Crimea. Int J Syst Evol Microbiol 55:1817-1826

Hou S, Makarova KS, Saw JH et al (2008) Complete genome sequence of the extremely acidophilic methanotroph isolate V4, Methylacidiphilum infernorum, a representative of the bacterial phylum Verrucomicrobia. Biol Direct 3:26

Hugenholtz P, Goebel BM, Pace NR (1998) Impact of culture-independent studies on the emerging phylogenetic view of bacterial diversity. J Bacteriol 180:4765-4774

Iguchi H, Yurimoto H, Sakai Y (2011) Methylovulum miyakonense gen. nov., sp. nov., a type I methanotroph isolated from forest soil. Int J Syst Evol Microbiol 61:810-815

Islam T, Jensen S, Reigstad LJ et al (2008) Methane oxidation at 55 degrees $\mathrm{C}$ and pH 2 by a thermoacidophilic bacterium belonging to the Verrucomicrobia phylum. Proc Natl Acad Sci USA 105:300-304

Jacob DJ (1999) Introduction to atmospheric chemistry. Princeton University Press, Princeton, USA. isbn:0-691-00185-5

Keltjens JT, Pol A, Reimann J et al (2014) PQQ-dependent methanol dehydrogenases: rare-earth elements make a difference. Appl Microbiol Biotechnol 98:6163-6183

Khadem AF, Pol A, Jetten MSM et al (2010) Nitrogen fixation by the verrucomicrobial methanotroph "Methylacidiphilum fumariolicum" SolV. Microbiology 156:1052-1059

Khadem AF, Pol A, Wieczorek A et al (2011) Autotrophic methanotrophy in verrucomicrobia: Methylacidiphilum fumariolicum SolV uses the Calvin-Benson-Bassham cycle for carbon dioxide fixation. J Bacteriol 193:4438-4446

Khadem AF, Pol A, Wieczorek A et al (2012a) Metabolic regulation of "Ca. Methylacidiphilum fumariolicum" SolV cells grown under different nitrogen and oxygen limitations. Front Microbiol 3:266

Khadem AF, van Teeseling MC, van Niftrik L et al (2012b) Genomic and physiological analysis of carbon storage in the verrucomicrobial methanotroph "Ca. Methylacidiphilum fumariolicum" SolV. Front Microbiol 3:345

Knittel K, Boetius A (2009) Anaerobic oxidation of methane: progress with an unknown process. Annu Rev Microbiol 63:311-334

Kozubal MA, Macur RE, Jay ZJ et al (2012) Microbial iron cycling in acidic geothermal springs of Yellowstone National Park: integrating molecular surveys, geochemical processes, and isolation of novel Fe-active microorganisms. Front Microbiol 3:109 
Li J, Peng X, Zhang L et al (2016) Linking microbial community structure to S, N and Fe biogeochemical cycling in the hot springs at the Tengchong geothermal fields, Southwest China. Geomicrobiol J 33:135-150

Linton JD, Cripps RE (1978) Occurrence and identification of intracellular polyglucose storage granules in Methylococcus NCBI 11083 grown in chemostat culture on methane. Arch Microbiol 117:41-48

Milucka J, Ferdelman TG, Polerecky L et al (2012) Zero-valent sulphur is a key intermediate in marine methane oxidation. Nature 491:541-546

Mohammadi S, Pol A, van Alen TA (2017) Methylacidiphilum fumariolicum SolV, a thermoacidophilic 'Knallgas' methanotroph with both an oxygen-sensitive and -insensitive hydrogenase. ISME J 11:945-958

Moss A, Jouany JP, Newbold J (2000) Methane production by ruminants: its contribution to global warming. Ann Zootech 49:231-253

Nguyen HH, Elliott SJ, Yip JH et al (1998) The particulate methane monooxygenase from Methylococcus capsulatus (Bath) is a novel copper-containing three-subunit enzyme. J Biol Chem 273: 7957-7966

Op den Camp HJM, Islam T, Stott MB et al (2009) Environmental, genomic and taxonomic perspectives on methanotrophic Verrucomicrobia. Environ Microbiol Rep 1:293-306

Pagaling E, Yang K, Yan T (2014) Pyrosequencing reveals correlations between extremely acidophilic bacterial communities with hydrogen sulphide concentrations, $\mathrm{pH}$ and inert polymer coatings at concrete sewer crown surfaces. J Appl Bacteriol 117:50-64

Pieja AJ et al (2011a) Distribution and selection of poly-3-hydroxybutyrate production capacity in methanotrophic Proteobacteria. Microb Ecol 62:564-573

Pieja AJ et al (2011b) Poly-3-hydroxybutyrate metabolism in the Type II methanotroph Methylocystis parvus OBBP. Appl Environ Microbiol 77:6012-6019

Pol A, Heijmans K, Harhangi HR et al (2007) Methanotrophy below pH 1 by a new Verrucomicrobia species. Nature 450:874-878

Pol A, Barends TR, Dietl A et al (2014) Rare earth metals are essential for methanotrophic life in volcanic mudpots. Environ Microbiol 16:255-264

Raghoebarsing AA, Pol A, van de Pas-Schoonen KT et al (2006) A microbial consortium couples anaerobic methane oxidation to denitrification. Nature 440:918-921

Rasigraf O, Kool DM, Jetten MSM et al (2014) Autotrophic carbon dioxide fixation via the CalvinBenson-Bassham cycle by the denitrifying methanotroph "Candidatus Methylomirabilis oxyfera". Appl Environ Microbiol 80:2451-2460

Scheller S, Yu H, Chadwick GL et al (2016) Artificial electron acceptors decouple archaeal methane oxidation from sulfate reduction. Science 351:703-707

Sharp CE, Stott MB, Dunfield PF (2012) Detection of autotrophic verrucomicrobial methanotrophs in a geothermal environment using stable isotope probing. Front Microbiol 3:303

Sharp CE, den Camp HJM O, Tamas I et al (2013) Unusual members of the PVC superphylum: the methanotrophic Verrucomicrobia genus "Methylacidiphilum". In: Fuerst JA (ed) Planctomycetes: cell structure, origins and biology. Springer-Verlag, Berlin, pp 211-227

Sharp CE, Smirnova AV, Graham JM et al (2014) Distribution and diversity of Verrucomicrobia methanotrophs in geothermal and acidic environments. Environ Microbiol 16:1867-1878

Šmejkalová H, Erb TJ, Fuchs G (2010) Methanol assimilation in Methylobacterium extorquens AM1: demonstration of all enzymes and their regulation. PLoS One 5:e13001

Strong PJ, Xie S, Clarke WP (2015) Methane as a resource: can the methanotrophs add value? Environ Sci Technol 49:4001-4018

Tavormina PL, Orphan VJ, Kalyuzhnaya MG et al (2011) A novel family of functional operons encoding methane/ammonia monooxygenase-related proteins in gammaproteobacterial methanotrophs. Environ Microbiol Rep 3:91-100

Tchawa Yimga M, Dunfield PF, Ricke P et al (2003) Wide distribution of a novel pmoA-like gene copy among type II methanotrophs, and its expression in Methylocystis strain SC2. Appl Environ Microbiol 69:5593-5602 
Thauer RK (1998) Biochemistry of methanogenesis: a tribute to Marjory Stephenson. Microbiology 144:2377-2406

van Teeseling MCF, Pol A, Harhangi HR et al (2014) Expanding the verrucomicrobial methanotrophic world: description of three novel species of Methylacidimicrobium gen. nov. Appl Environ Microbiol 80:6782-6791

Vekeman B, Kerckhof FM, Cremers G et al (2016) New Methyloceanibacter diversity from North Sea sediments includes methanotroph containing solely the soluble methane monooxygenase. Environ Microbiol 18:4523-4536

Vernikos GS, Parkhill J (2006) Interpolated variable order motifs for identification of horizontally acquired DNA: revisiting the Salmonella pathogenicity islands. Bioinformatics 22:2196-2203

Wagner M, Horn M (2006) The Planctomycetes, Verrucomicrobia, Chlamydiae and sister phyla comprise a superphylum with biotechnological and medical relevance. Curr Opin Biotechnol 17:241-249 